\title{
An IgM capture enzyme linked immunosorbent assay to detect IgM antibodies to treponemes in patients with syphilis
}

\author{
O E IJSSElMUIDEN,* J J van der Sluis, * A MUlder, $\dagger$ E STOLZ,* K P BOLTON, \\ R V W VAN EIJK $†$
}

From the *Department of Dermatovenereology, University Hospital Dijkzigt, Rotterdam, the $\nmid$ Laboratory for Bacteriology, National Institute of Public Health and Environmental Protection, Bilthoven, The Netherlands, and $\ddagger$ Department of Paediatrics, Baragwanath Hospital, Johannesburg, South Africa

SUMMARY A new IgM capture enzyme linked immunosorbent assay (ELISA) was compared with the 19S(IgM) fluorescent treponemal antibody absorption (19S(IgM)FTA-ABS) test for detecting IgM antibodies to treponemes. Serum samples from 180 people, 109 with various stages of untreated syphilis, 45 with treated syphilis, and 26 non-infected, were investigated.

In all diagnostic groups of syphilis the reactivity of the IgM capture ELISA was similar to that of the 19S(IgM)FTA-ABS test except in untreated neurosyphilis, for which the IgM capture ELISA was significantly less sensitive. The IgM capture ELISA was very sensitive in congenital $(100 \%, 5 / 5)$ and primary $(82 \%, 18 / 22)$ syphilis, but less sensitive in secondary $(60 \%, 12 / 20)$, latent $(53 \%, 16 / 30)$, neurosyphilis $(34 \%, 11 / 32)$, and treated $(11 \%, 5 / 45)$ syphilis.

False positive IgM capture ELISA results were not found in five people who gave false positive Venereal Disease Research Laboratory (VDRL) reactions or in 21 neonates born to mothers adequately treated for syphilis before or during pregnancy. This indicated that the IgM capture ELISA was very specific.

The course of antitreponemal IgM reactivity after treatment of early infectious syphilis was followed up in six patients. The quantity of $\operatorname{IgM}$ antibody declined in nearly all patients after treatment, but still remained detectable in five patients up to six months after treatment. In contrast, non-treponemal antibodies measured by the VDRL test disappeared in four out of six patients within five months from starting treatment.

In conclusion, the IgM capture ELISA may be useful for easy and sensitive detection of IgM antibodies to treponemes in patients with congenital and primary syphilis. A positive test result in these cases indicates that patients should receive treatment if they have not been treated recently. The test is not, however, recommended to replace the VDRL test to monitor patients treated for syphilis.

IgM class antibodies to treponemes are usually the first to be produced during treponemal infection. IgG class antibodies appear rapidly thereafter, and soon become predominant.' Testing for antitreponemal IgM has been thought to be useful for detecting active disease early, ${ }^{2}$ evaluating treatment, ${ }^{13}$ and screening for congenital syphilis in neonates. ${ }^{4}$

Address for reprints: Dr R V W van Eijk, Central Laboratory of The Netherlands Red Cross Blood Transfusion Service, PO Box 9190, 1006 AD Amsterdam, The Netherlands

Accepted for publication 21 August 1988
Some investigators ${ }^{25}$ have indicated a need to separate the IgM fraction from serum before testing, to avoid false negative results caused by IgG masking IgM and to avoid false positive results caused by rheumatoid factor. Several methods to accomplish this have been described. They include ultracentrifugation, ${ }^{1}$ ion exchange, ${ }^{6}$ size exclusion, ${ }^{5}$ and affinity chromatography. ${ }^{6}$ These techniques are used to separate antitreponemal IgM before testing by immunofluorescence or haemagglutination.

In The Netherlands the 19S(IgM) fluorescent treponemal antibody absorption (FTA-ABS) test, using size exclusion chromatography to isolate IgM 
antibodies, is generally accepted as the standard procedure for detecting antitreponemal IgM. Application of this test is, however, limited by the need for special equipment and by technical complexity. Technically simpler methods suitable for detecting antitreponemal IgM routinely have been proposed. They include the IgM solid phase haemadsorption assay (SPHA), ${ }^{7}$ the enzyme linked immunosorbent assay (ELISA) to detect antiaxial filament IgM (IgMELISA), ${ }^{2}$ and the Treponema pallidum specific IgM haemagglutination assay (TP-IgM-HA) ${ }^{8}$ Because of doubts raised about the sensitivity of the SPHA ${ }^{9}$ and the lack of commercially available reagents for the IgM-ELISA and the TP-IgM-HA, these tests have never been generally accepted as suitable for detecting IgM antibodies to treponemes.

In the present study we compared a recently introduced IgM capture ELISA (Captia Syphilis-M; Mercia Diagnostics, Guildford, England) with the 19S(IgM)FTA-ABS test to detect IgM antibodies to treponemes in patients with treated or untreated syphilis.

\section{Patients, materials, and methods}

SERUM SAM PLES

Serum samples from 154 patients with syphilis were obtained from the sexually transmitted disease clinic at the University Hospital, Rotterdam, and the Department of Neurology, the Free University, Amsterdam. One hundred and nine of these patients were untreated. The samples from the 45 patients treated for syphilis were collected at various intervals after the start of treatment. The diagnosis and classification of syphilis was made according to criteria described previously. ${ }^{10}$ All samples were reactive in at least the FTA-ABS test.

Consecutive samples from five patients with primary syphilis and one patient with early latent syphilis, which were collected at the start of treatment and at various intervals after treatment, were used to evaluate IgM antibody to treponemes after treatment. The samples were tested in the same run by the IgM capture ELISA and the Venereal Disease Research Laboratory (VDRL) test, and on the same day by the 19S(IgM)FTA-ABS test. The serum samples of all patients were reactive in the FTA-ABS and VDRL tests at the start of treatment. In addition samples from five patients with solitary false positive VDRL test reactions were used. Samples from five neonates with clinical signs indicative of congenital syphilis were obtained from the Baragwanath Hospital in Johannesburg. Samples giving positive results in the $T$ pallidum haemagglutination assay (TPHA) and the FTA-ABS test were obtained from 21 neonates without clinical signs indicative of syphilis who attended
Ijsselmuiden, van der Sluis, Mulder, Stolz, Bolton, Eijk

various clinics in The Netherlands.

All serum samples were tested using the TPHA (Japan Lyophilisation Company, Japan), the FTAABS test, "the 19S(IgM)FTA-ABS test, the VDRL test," and the antitreponemal IgM capture ELISA. Rheumatoid factor was detected by a latex agglutination test (Rapitex, Behring Werke, West Germany). All samples were stored at $-20^{\circ} \mathrm{C}$.

\section{S(IgM)FTA-ABS test}

IgM was separated by gel filtration chromatography using high performance liquid chromatography (HPLC; LKB, Sweden). A $50 \mu$ l volume of serum, prefiltered through a $0.2 \mu \mathrm{m}$ filter (Millex G-V, Millipore), was passed through a $7.5 \times 75 \mathrm{~mm}$ column (TSK GSWP; Toya Soda, Japan) that had been equilibrated with $0.25 \mathrm{mmol} / \mathrm{l}$ phosphate buffered

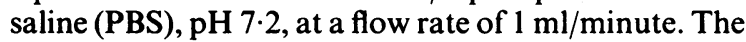
eluate was monitored at $280 \mathrm{~nm}$, and fractions of $50 \mu \mathrm{l}$ were collected. The IgM fraction was partially excluded by the gel. The mean IgG contamination in the fraction containing IgM was less than $25 \mathrm{mg} / \mathrm{l}$ and the molar IgM/IgG ratio was 200 -fold greater in favour of IgM than before fractionation. The IgM was diluted seven times. Concentrations of IgM and IgG were measured by a fluoroimmunoassay. ${ }^{12}$

The IgM fractions were investigated with an indirect immunofluorescent assay according to the protocol of the FTA-ABS test." To minimise the risk of false positive and negative test results because of contaminating IgG, IgG was investigated separately using the FTA test with the appropriate anti-IgG conjugate. In all cases except one, as indicated, no fluorescence was observed.

\section{Antitreponemal IgM capture ELISA}

IgM antibodies to treponemes were detected by a capture ELISA procedure using test kits that included microtitration plates coated with antihuman IgM, positive and negative control serum samples, dilution buffer, wash buffer, tracer complex, and substrate. The test was performed according to the manufacturer's instructions. Briefly, microtitre plate wells coated with rabbit antihuman IgM were incubated for one hour at $37^{\circ} \mathrm{C}$ with $100 \mu \mathrm{l}$ serum diluted $1 / 50$ in dilution buffer. Control serum samples (positive at high and low titres and negative) were included in every experiment. After the wells had been emptied and washed five times with $300 \mu \mathrm{l}$ wash buffer, the plates were incubated for one hour with $100 \mu$ l tracer complex diluted $1 / 20$. The tracer complex consisted of $T$ pallidum antigens, a biotinylated antiaxial filament monoclonal IgM antibody, and streptavidin conjugated with horseradish peroxidase (HRP). The monoclonal antibody appeared to be of the IgM class by radial immunodiffusion. The wells were washed again as mentioned 
above, and $100 \mu$ substrate was added. The reaction was stopped after 30 minutes by adding $25 \mu \mathrm{l}$ of $2 \mathrm{~mol} / \mathrm{l}$ sulphuric acid. The plates were read on a multiscan reader (Titertek) at $450 \mathrm{~nm}$. The cut off point between positive and negative was set at the absorbance of the low titre positive control according to the manual supplied by the manufacturer. Serum samples showing an absorbance within about $10 \%$ of the low titre positive control were tested again.

STATISTICAL ANALYSIS

The sign test was used to compare the results of the different tests.

Results

IGM ANTIBODIES TO TREPONEMES IN PATIENTS WITH UNTREATED SYPHILIS

Serum samples from 109 patients with untreated syphilis were investigated with the IgM capture ELISA and the results compared with those of the 19S(IgM)FTA-ABS test (table 1). Both tests showed comparable sensitivity at all stages of untreated syphilis except for neurosyphilis. The sensitivity of the IgM capture ELISA was high in congenital $(100 \%)$ and primary $(82 \%)$ syphilis, but less in secondary $(60 \%)$ and latent syphilis $(53 \%)$. In patients with neurosyphilis the reactivity of the IgM capture ELISA was significantly lower $(34 \%)$ than that of the $19 \mathrm{~S}$ (IgM)FTA-ABS test (72\%). Of the 12 samples from patients with neurosyphilis that were reactive in the 19S(IgM)FTA-ABS test but not the IgM capture ELISA, two had absorbance values within $10 \%$ below those of the low titre positive control. Retesting those samples did not alter the results. Fluorescence in the 19S(IgM)FTA-ABS test was strong in seven out of these 12 samples and weak in the remaining five. All 32 samples from patients with neurosyphilis gave negative results when tested for the presence of rheumatoid factor.

Table 1 IgM antibodies to Treponema pallidum in serum samples from 109 patients with untreated syphilis comparing results in IgM capture ELISA with those in 19S(IgM)FTAABS test

\begin{tabular}{lclll}
\hline $\begin{array}{l}\text { Diagnostic } \\
\text { category } \\
\text { of syphilis }\end{array}$ & No & ELISA & FTA-ABS & \begin{tabular}{l} 
Nercentage \\
\cline { 3 - 5 } agreement
\end{tabular} \\
\hline Primary & 22 & 18 & 18 & 100 \\
Secondary & 20 & 12 & 14 & 80 \\
Latent & 30 & 16 & 18 & 87 \\
Congenital & 5 & 5 & 5 & 100 \\
Neurosyphilis & 32 & 11 & 23 & 63 \\
\hline
\end{tabular}

ELISA = enzyme linked immunosorbent assay.

FTA-ABS = fluorescent treponemal antibody absorption (test).
IGM ANTIBODIES TO TREPONEMES IN PATIENTS WITH TREATED SYPHILIS AND NON-INFECTED PEOPLE

Table 2 shows the incidence of IgM antibodies to treponemes in 71 serum samples from patients with adequately treated syphilis, people showing false positive VDRL reactions, and non-infected neonates who had positive treponemal reactions. The patients with treated syphilis were divided into two categories; those with positive treponemal test results and a positive VDRL reaction, and those with only positive treponemal test results. Of the 23 samples from patients with treated syphilis who showed positive VDRL reactions, three were positive in both the IgM capture ELISA and the 19S(IgM)FTA-ABS test and two were each reactive in one test only. Of the 22 samples from treated patients with a negative VDRL reaction, only one gave a positive result in the IgM capture ELISA. No evidence of rheumatoid factor was found in any of these serum samples. IgM antibodies to treponemes were not found in the five people who had false positive VDRL reactions. The 21 serum samples from neonates of mothers adequately treated for syphilis before or during pregnancy also gave negative results in both IgM tests. All neonates, however, gave positive results in the TPHA and the FTA-ABS test.

\section{COURSE OF REACTIVITY TO IGM ANTIBODIES TO TREPONEMES AFTER TREATMENT}

To assess the influence of treatment on reactivity in the IgM capture ELISA, consecutive serum samples from six patients with early infectious syphilis were investigated for up to 10 months after the start of treatment. The figure compares the results of the IgM capture ELISA with those of the 19S(IgM)FTA-ABS and

Table 2 IgM antibodies to Treponema pallidum in serum samples from 45 patients with treated syphilis and 26 noninfected people comparing results in IgM capture ELISA with those in $19 S(\operatorname{IgM}) F T A-A B S$ test

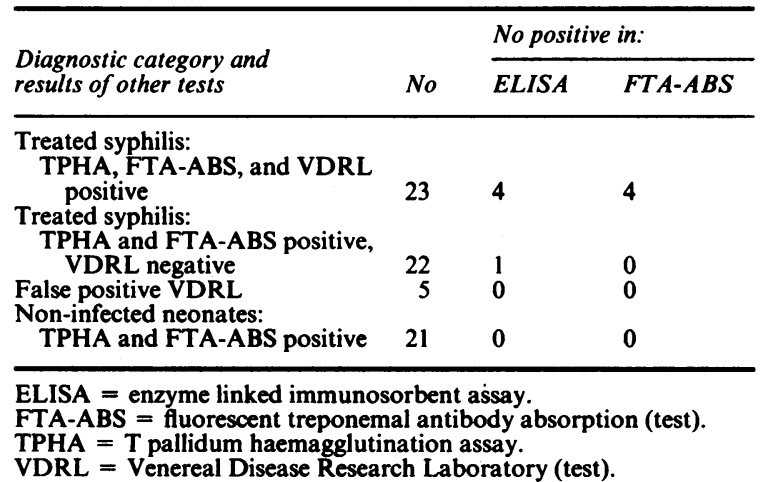



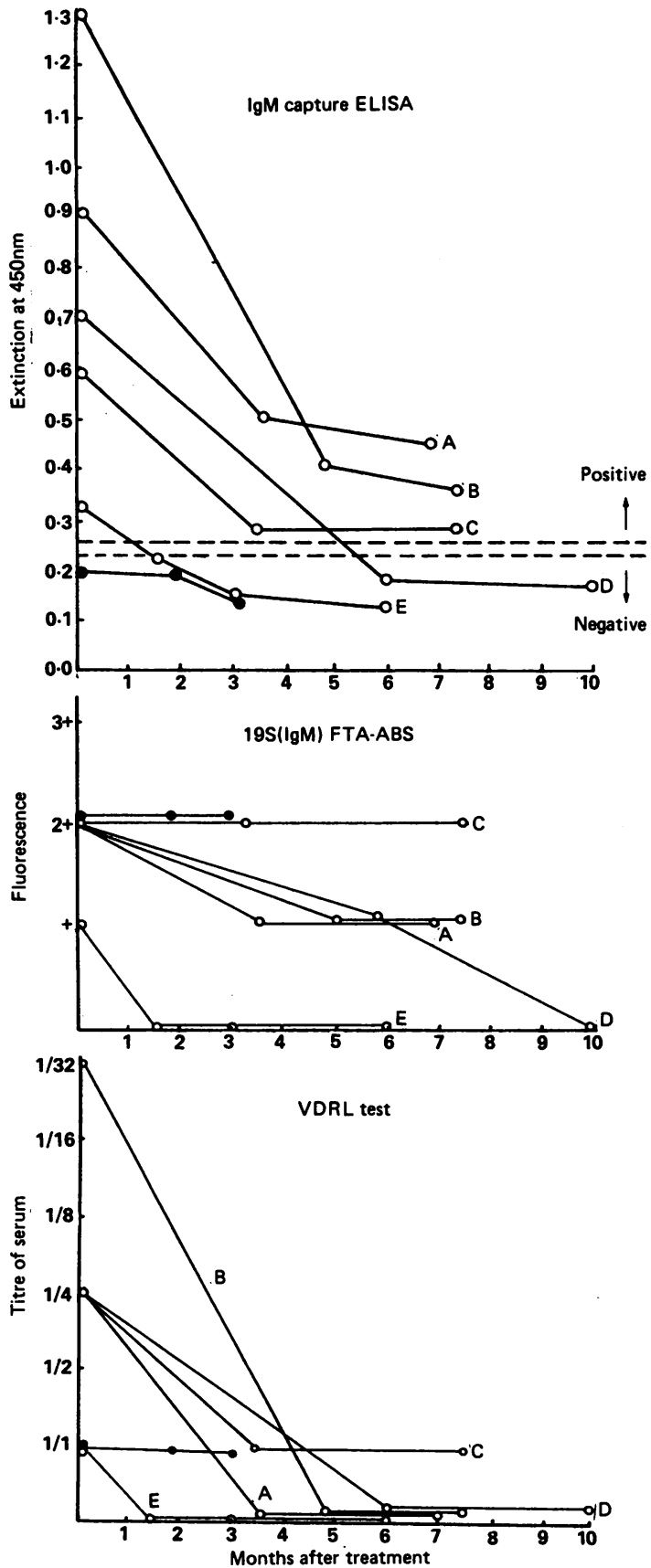

Figure Course of IgM reactivity in the IgM capture enzyme linked immunosorbent assay (ELISA), the 19S(IgM) fluorescent treponemal antibody absorption (FTA-ABS) test, and the Venereal Disease Research Laboratory (VDRL) test after treating five patients $(A-E)$ with primary and one patient (-) with early latent syphilis.
VDRL tests at various intervals after the start of treatment.

In all patients with primary syphilis the titre of IgM in both tests for IgM antibodies to treponemes declined as a result of treatment. After six months, however, three out of the six patients still gave positive IgM capture ELISA results and four gave positive 19S(IgM)FTA-ABS test results. All five patients with primary syphilis showed a positive VDRL reaction at the start of treatment, but five months after treatment only one still showed a positive result. A patient suffering from early latent syphilis was followed up for three months after treatment and was found to give negative results in the IgM capture ELISA and positive results in both the 19S(IgM)FTA-ABS and VDRL tests.

\section{Discussion}

Treponeme specific IgM can most reliably be detected in the 19S(IgM) fraction after its separation from serum. ${ }^{257-9}$ The $19 \mathrm{~S}(\mathrm{IgM}) \mathrm{FTA}$-ABS.test is widely used to show treponeme specific IgM, but the time consuming operation of this method makes a more simple procedure desirable.

The IgM capture ELISA shares with other recently developed tests the specific procedure of IgM immune adsorption (the binding of IgM antibodies to a solid phase that has been coated previously with antibodies to IgM raised in animals. ${ }^{13}$ However, detecting immune adsorbed IgM antibodies specific to treponemes using red blood cells sensitised to $T$ pallidum antigens ${ }^{7}$ or by ELISA ${ }^{2}$ may be insensitive. ${ }^{13}$ Although detecting adsorbed IgM by sensitised red blood cells is very specific, this technique is insensitive because the blood cells adhere insufficiently in the haemadsorption step..$^{913}$ In contrast to the SPHA, high sensitivity can be expected in the IgM capture ELISA because of the separation of IgM from IgG and the use of a biotinavidin complex, which amplifies the reaction of antigen with antibody.

Regarding the specificity of a capture ELISA used to detect IgM, rheumatoid factor can cause false positive results because, bound to the anti-IgM antibodies coated to the solid phase, rheumatoid factor can directly bind the crystallisable fragment of the IgG class conjugate. ${ }^{214}$ In the IgM capture ELISA false positive results are not likely to occur in this way because a tracer complex is used instead of an IgG class conjugate.

In early stages of syphilis the sensitivity of the IgM capture ELISA was $93 \%$ compared with the 19S(IgM)FTA-ABS test. In the case of neurosyphilis, however, the sensitivity of the IgM capture ELISA only reached $47 \%$ compared with that of the $19 \mathrm{~S}$ (IgM)FTA-ABS test. In addition, the consecutive 
serum samples from one patient with latent syphilis, which were collected at the onset of treatment and thereafter, did not react in the IgM capture ELISA although they showed strong fluorescence in the 19S(IgM)FTA-ABS test. Several explanations may be relevant for the differences in the sensitivity of the two tests in the patient with latent syphilis and those with neurosyphilis. Firstly, the immune response in the patients with latent syphilis could be directed against a limited range of $T$ pallidum antigens. If this range did not include the $T$ pallidum axial filament (32-39 kilodalton) components, to which the monoclonal antibody used in the IgM capture ELISA was directed, negative results could be expected. However, western blot analysis of IgM fractions from this patient did not show a different reaction pattern in the 32-39 kilodalton antigen range from that of a patient suffering from primary syphilis (results not shown). Performing the IgM capture ELISA on these serum samples using a monoclonal antibody directed to the 47 kilodalton antigen, which had been labelled by the manufacturer under the same conditions as the monoclonal antibody to axial filament, did not give positive reactions either. Secondly, the difference in sensitivity of the IgM capture ELISA and the 19S(IgM)FTA-ABS test could be.due to the fact that some epitopes of $T$ pallidum antigens first become immunogenic in late syphilis and that IgM antibodies raised to these epitopes may be detected in a immunofluorescence test and not in an ELISA, as has been pointed out by Pedersen et $_{\text {al. }}{ }^{2}$

In our hands the IgM capture ELISA was less successful than the currently used VDRL test in monitoring the effect of treatment. We do not know the reason for this, but the serum of treated patients with syphilis may contain $8 \mathrm{~S}(\mathrm{IgM})$ treponemal antibodies long after 19S(IgM) antibodies have disappeared. ${ }^{13}$ Detecting $8 \mathrm{~S}(\mathrm{IgM})$ antibodies by an IgM detection test may therefore mask the success of treatment as indicated by the absence of 19S(IgM) antibodies. The IgM capture ELISA probably cannot distinguish between 19S(IgM) and 8S(IgM) antibodies, ${ }^{13}$ and therefore serum samples from which 19S(IgM) antibodies are absent but that still contain 8S(IgM) antibodies may react in the IgM capture ELISA.

In conclusion, the IgM capture ELISA in its present form can be a useful test in early stages of syphilis and for screening for congenital syphilis. It might, however, be possible to improve the test for late syphilis, especially neurosyphilis, by setting the cut off point between positive and negative results to a lower value. Treatment monitoring with the IgM capture ELISA is not very useful because IgM antibodies to treponemes can be detected for longer after treatment than non-treponemal antibodies reactive in the VDRL test. The VDRL test is therefore able to detect the response to treatment more rapidly than a test detecting treponemal IgM. Finally, the absence of IgM antibodies to treponemes does not imply that syphilis is absent. $^{2}$

We thank $G$ Aelbers and $R$ van Niekerk for performing the many 19S(IgM)FTA-ABS tests and T Olij for typing help.

\section{References}

1 Shannon R, Booth S. The pattern of immunological response at various stages of syphilis. British Journal of Venereal Diseases 1977;53:281-6.

2 Strandberg Pedersen N, Sand Petersen C, Axelsen NH. Enzymelinked immunosorbent assay for detection of immunoglobulin $\mathbf{M}$ antibody against the Reiter treponeme flagellum in syphilis. J Clin Microbiol 1982;16:608-14.

3 Müller F, Wollemann G. Analysis of specific immunoglobulin M immune response to Treponema pallidum before and after penicillin treatment of human syphilis. European Journal of Sexually Transmitted Diseases 1985;2:67-72.

4 Johnston NA. Neonatal congenital syphilis. Diagnosis by the absorbed fluorescent treponemal antibody (IgM) test. British Journal of Venereal Diseases 1972;48:464-9.

5 Müller F. Der 19S(IgM)-FTA-ABS-test in der Serodiagnostik der Syphilis. Technik, Fehlermöglichkeiten und diagnostische Aussage. Immunitaet und Infektion 1982;10:23-34.

6 Huschka U, Stengel HH, Schroeter R, Roelcke D, Doerr HW. Rapid detection of Treponema pallidum and cytomegalovirus specific IgM antibodies with the passive haemagglutination. Zentralbl Bakteriol Mikrobiol Hyg [A] 1982;253:120-30.

7 Schmidt BL. Solid phase hemadsorption: a method for rapid detection of Treponema pallidum-specific IgM. Sex Transm Dis 1980;7:53-8.

8 Sato T, Kubo E, Yokota M, Kayashima T, Tomizawa T. Treponema pallidum specific IgM haemagglutination test for serodiagnosis of syphilis. British Journal of Venereal Diseases 1984;60:364-70.

9 Eichmann A, Götling M, Meyer JCh. The SPHA test in the diagnosis of syphilis-results in various stages of untreated syphilis. European Journal of Sexually Transmitted Diseases 1986;3:95-8.

10 van Eijk RVW, Menke HE, Tideman GJ, Stolz E. Enzyme linked immunosorbent assays with Treponema pallidum or axial filament of $T$ phagedenis biotype Reiter as antigen: evaluation as screening tests for syphilis. Genitourin Med 1986;62:367-72.

11 US Public Health Service. Manual of tests for syphilis. Washington DC: US Department of Health, Education and Welfare, US Government Printing Office, 1969 (Public Health Service Publication No 411): 1-77.

12 Hische EAH, van der Helm HJ, Out T. Estimation of IgM in cerebrospinal fluid by fluoroimmunoassay. Clin Chim Acta 1979;97:93-5.

13 Müller F, Lindenschmidt E-G. Demonstration of specific 19S(IgM) antibodies in untreated and treated syphilis. British Journal of Venereal Diseases 1982;58:12-7.

14 Naot Y, Barnett EV, Remington JS. Method for avoiding falsepositive results occurring in immunoglobulin $M$ enzyme-linked immunosorbent assays due to presence of both rheumatoid factor and antinuclear antibodies. J Clin Microbiol 1981;14: 73-8. 